\title{
Prevención del cáncer cérvico-uterino en Chile. Mucha vacuna y poco Papanicolau
}

\begin{abstract}
Alberto Fica
Hospital Militar de Santiago, Chile.

Departamento de Medicin Servicio de Infectología.

Conflicto de interés: ninguno Financiamiento: ninguno

Recibido: 24 de enero de 2014 Aceptado: 6 de febrero de 2014

Correspondencia a: Alberto Fica Cubillos albertofica@gmail.com

Cancer of cervix in Chile. Too much vaccine amid a neglected Papanicolau

The Chilean Ministry of Health announced the incorporation of a human papillomavirus (HPV) vaccine to prevent cervix uterine cancer (CUC) into the national immunization program during year 2014 This decision was adopted despite of two opposing documents and a significant decrease in cervical cancer associated mortality due to cytological cervical screening. The burden of disease attributed to CUC has declined in Chile and current costeffectiveness studies should be reviewed considering this decreasing trend, the progressive decrease in coverage rates observed during the past years, the potential need for aditional doses and lower vaccine costs if vaccine is acquired through the PAHO revolving fund. Moreover, serious adverse events associated with these vaccines, which in some countries are more frequent than CUC associated mortality, have not been thoroughly evaluated and are probably underreported. The decision to incorporate the vaccine occurs in a context of progressive weakening of the national cervical screening program leading to a reduced population coverage. This situation jepeordizes the achievements already obtained and poses a challenge to vaccine introduction considering that not all the high-risk viral subtypes are included and thus the risk for CUC does not disappear making cervical screening a vital component of the program that needs to be maintained. This governmental resolution requires a more solid scientific foundation and should not be implemented without resolving current cervical screening shortcomings.

Key words: Uterine cervix cancer, early detection of cancer, papillomavirus vaccines, cost effectiveness.

Palabras clave: Cáncer cérvico-uterino, detección precoz del cáncer, vacunas para virus papiloma, costoefectividad.
\end{abstract}

Réplica de MINSAL en pág. 204

L a reciente decisión del Ministerio de Salud de Chile de universalizar e incorporar la vacuna para virus papiloma humano (VPH) en el Programa Nacional de Inmunizaciones desde el año 2014 parece ser un importante paso en salud pública destinado a la prevención del cáncer cérvico-uterino (CCU) y que justificaría el gasto de importantes fondos con este laudable propósito. El contar con una herramienta de prevención primaria de este cáncer que podría reducir las muertes y el sufrimiento asociado a él, pareciera ser una mejor alternativa que la prevención secundaria lograda con el clásico método citológico del Papanicolau.

Esta decisión fue tomada a pesar de existir dos documentos discrepantes emanados del propio Ministerio o de instancias asesoras del Ministerio en años recientes, uno que la encontraría infundada y otro que la respaldaría ${ }^{1,2}$. Estas diferencias fueron expresadas también hace cinco años entre el Comité Consultivo de Inmunizaciones de la Sociedad Chilena de Infectología e integrantes del mundo académico con miradas discrepantes ${ }^{3-5}$. Es por esto y por otras interrogantes que esta decisión merece ser comentada y analizada ampliamente.
El cáncer cérvico-uterino (CCU) representaba en los años 90 la tercera causa de cáncer en el mundo en mujeres, además de ser la primera causa de muerte por cáncer en este género, afectando principalmente a países en vías de desarrollo con $78 \%$ del total de las muertes (cerca de 190.000 muertes al año) ${ }^{6}$. No obstante, ya en esa época, la tasa cruda de mortalidad en los países del cono sur, incluyendo Chile, era inferior a muchas otras regiones del mundo, con cifras equivalentes a los países de Europa del Este y levemente superiores a los países del norte y oeste de Europa (10,6 vs 7,5-7,7 por 100.000 mujeres en este último caso $)^{6}$. La tasa máxima de incidencia de este cáncer ocurre entre los 55 y 64 años de edad en países en desarrollo y unos 10 años después en países desarrollados? El CCU tiene una mortalidad significativa en casos de diagnóstico tardío y hacia el año 2010, moría más de una mujer al día en Chile por esta causa (aproximadamente 580 al año) ${ }^{8}$. El CCU es una de las principales causas de pérdida de vida de años saludables en mujeres ya que las afecta en etapa fértil y laboralmente productiva.

El CCU es provocado por la presencia del virus papiloma humano (VPH) que es un factor necesario pero 
no suficiente, ya que cerca de $90 \%$ de las infecciones cervicales por VPH se resuelven espontáneamente dentro de dos años; sólo una fracción se asocia a una infección persistente que puede llevar, luego de varios años, en caso de subtipos de alto riesgo, a la displasia del epitelio del cuello uterino y progresar en algunos casos a lesiones pre-cancerosas (neoplasia intra-epitelial NIE grados II o III) o CCU9 . Incluso, pacientes con neoplasias intraepiteliales grados II o III pueden tener lesiones regresivas o no progresivas que no necesariamente evolucionarán a cáncer invasor ${ }^{9}$.

Este virus pertenece a una familia que comprende más de 90 subtipos que son clasificados de acuerdo a su potencial oncogénico en aquellos de alto riesgo oncogénico, de bajo riesgo y de riesgo potencial o probable. Cerca de 30 subtipos del VPH aparecen involucrados en lesiones pre-cancerosas invasoras o pre invasoras anales o genitales secundarias a la adquisición de los virus durante diferentes formas de actividad sexual ${ }^{3,7}$. A pesar de que sólo 8 subtipos serían causales de $90 \%$ de los casos de CCU: $16,18,45,33,31,52,58$ y 35, los subtipos 16 y 18 explicarán aproximadamente el $70 \%$ de los casos de $\mathrm{CCU}^{9}$. Además, una proporción variable de casos de cáncer de vulva, vagina, ano, pene y orofaringe también serían causados por la infección con subtipos de VPH de alto riesgo ${ }^{3}$. La participación de los VPH en tumorogénesis se extiende también a las verrugas genitales donde la mayor parte de ellas se asocia a los subtipos 6 y $11^{3}$. Como se mencionó previamente, la mayor parte de las mujeres que adquieren la infección están libres de ella al cabo de dos años ${ }^{9}$.

La disminución mundial de la mortalidad por $\mathrm{CCU}$ se ha logrado por programas de tamizaje con citología cervical que detecta lesiones pre-cancerosas o cancerosas a través del Papanicolau y ahora se ha introducido a este método la detección del virus con herramientas de biología molecular como RPC e hibridación cuyo aporte en la prevención del CCU aún está en definición y en activo debate $^{10-12}$. El tamizaje con la técnica del Papanicolau permite la pesquisa precoz del CCU y su tratamiento en etapas iniciales resultando en altas tasas de curación y una mortalidad reducida.

Luego de iniciar la pesquisa de CCU en Chile el año 1965, nuestro país reconfiguró su programa de CCU el año 1987 convirtiendo el tamizaje por oportunidad en mujeres jóvenes hacia un programa sistemático focalizado a mujeres de alto riesgo de CCU (entre 25 y 64 años). Este programa incluía al menos un examen cada tres años, acceso a terapia de las lesiones pre-cancerosas o el cáncer con cobertura financiera, control de calidad de la citología de las muestras cervicales, creación de un laboratorio nacional de referencia de citología cervical, educación al personal de salud y campañas educativas a la población susceptible ${ }^{13,14}$.

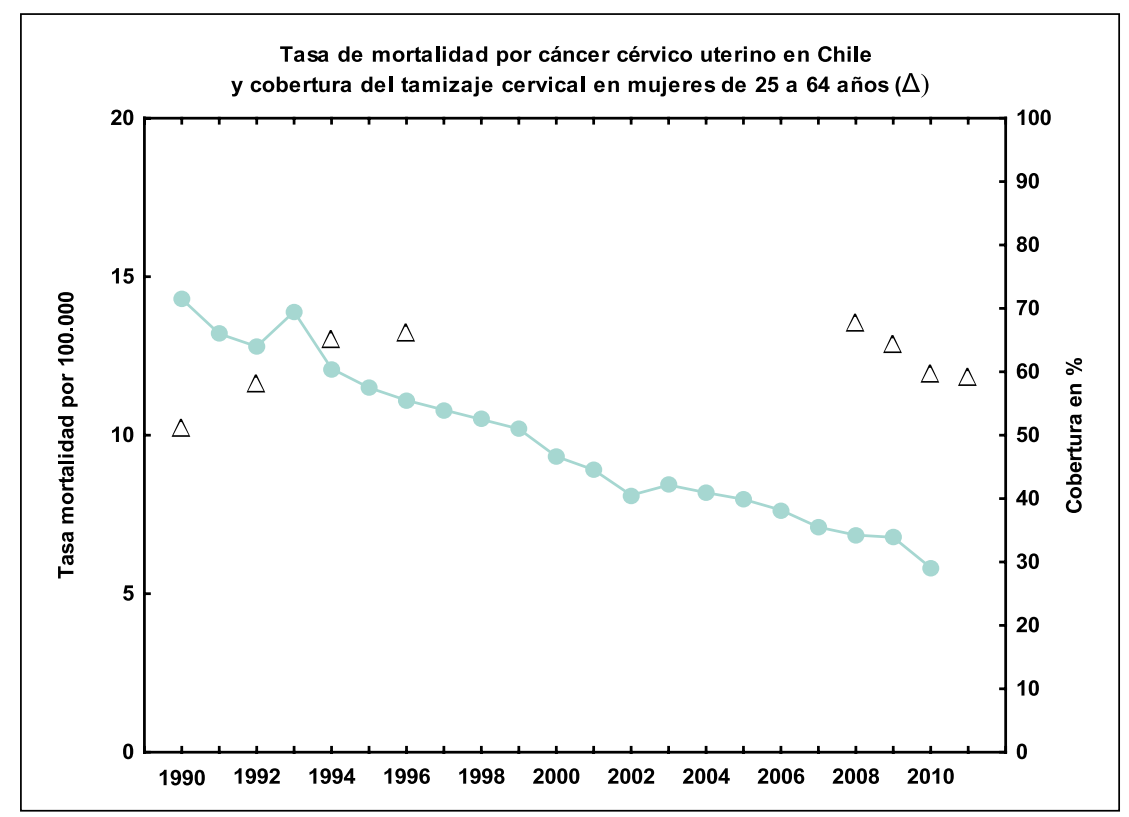

Figura 1. Tasa de mortalidad por CCU en Chile y cobertura del tamizaje cervical en mujeres de 25 a 64 años. Los datos fueron extraídos y adaptados de las referencias 8,13 y 15.

El impacto de este programa fue dramático, con un aumento progresivo de las coberturas del tamizaje y una reducción paralela de la mortalidad $\mathrm{CCU}$ en los años siguientes. Así, la cobertura aumentó desde $51 \%$ en el año 1990 a $66 \%$ en 1996 y siguientes, y la tasa de mortalidad ajustada descendió desde 14,3 en 1990 a 8,5 por 100.000 en el 2003, representado esta última un $40 \%$ menos de casos (Figura 1) ${ }^{14-16}$. El aumento de la cobertura significó incrementar el porcentaje o fracción de la detección de lesiones de alto grado sobre los casos que ya tenían carcinoma cervical in situ, permitiendo así tratamientos curativos $^{14-16}$.

El aumento de la cobertura en el tamizaje parece depender de varios factores estratégicos que incluyen el compromiso de los servicios de salud locales, programas computacionales específicos con retro-alimentación estadística, incorporación de la comunidad al programa y capacitación del personal de salud ${ }^{17}$. Durante la implementación de este programa fue evidente la existencia de barreras culturales para la realización del tamizaje en la población lo que requirió un gran esfuerzo por los equipos de salud y un ajuste en la forma de acercamiento y trabajo comunitario $^{18,19}$. Desafortunadamente, la cobertura del tamizaje nunca alcanzó llegar al ideal 80\%, meta indicada en el programa y tomada de la experiencia sobre este problema en países nórdicos. Los mejores porcentajes nacionales no superaron el $67 \%$ y en los últimos años se observa un grave deterioro en la cobertura llegando a un promedio de 59\% el año 2011 (Figura 1). Este problema 
se asoció a limitaciones financieras sustantivas y evidentes a comienzos de la década del $2000^{14}$.

El tamizaje implica una consulta de salud que va acompañado de al menos otros tres beneficios, además de la pesquisa del CCU y que incluyen un acceso más igualitario a la salud, la pesquisa de infecciones de transmisión sexual y de otras patologías de la esfera ginecológica como el cáncer de mama, útero y ovarios y la educación para la prevención o pesquisa precoz de estos problemas.

Respecto a la equidad, las coberturas de tamizaje son más bajas en los pacientes del sub-sistema público de salud respecto a los del sub-sistema privado, siendo la diferencia al menos 10 puntos porcentuales más alta para las pacientes del sub-sistema privado en Chile ${ }^{13}$. Con el programa sistemático de CCU en Chile, las coberturas en el sub-sistema público subieron más que el alza observada en el sub-sistema privado, generando así una mayor equidad respecto de esta importante herramienta preventiva del $\mathrm{CCU}^{13}$. Auditorías de muerte por CCU revelaron claramente que cerca de la mitad de los decesos por esta causa se registraron en mujeres sin evidencias previas de actividades de pesquisa y se concentraron en comunas con la menor cobertura de tamizaje $\mathrm{e}^{20}$. En otras palabras, el deterioro actual en las coberturas del tamizaje cervical perjudica principalmente a las mujeres más pobres en Chile. En línea con este hecho, en varios países latinoamericanos como Bolivia, Brasil, Guatemala y República Dominicana, el tamizaje cervical es más probable en los quintiles más ricos de la población y en zonas urbanas respecto a las rurales ${ }^{21}$. Las causas de este deterioro en Chile residen básicamente en la falta de un programa efectivo de fomento comunicacional respecto del tamizaje y detección y además, en falencias en la atención primaria que es donde se efectúa el tamizaje, y esto pareciera acompañarse de otros retrocesos preventivos como la baja en el índice de pesquisa por tuberculosis. Este índice expresa el número de baciloscopias de expectoración por cada 1.000 consultas de adultos en atención primaria, siendo su óptimo 50 pero con cifras actuales a un $25 \%$ de ese valor, impidiendo así la identificación de casos de tuberculosis en la comunidad ${ }^{22}$. Estos datos insinúan que se debe investigar con mayor detalle las razones en la baja de las coberturas del tamizaje de CCU para evitar un impacto negativo en las tasas de mortalidad por esta causa en los años que vienen. Hacia el año 2007, a 20 años de iniciar el programa, Chile era considerado un país líder en el control del CCU entre los países en vías de desarrollo ${ }^{23}$. Algunas de las deficiencias del programa han sido ya identificadas e incluyen aspectos que no requieren de mayores aportes presupuestarios y en cambio otras sí. Entre las primeras se ha señalado la optimización de indicadores y registros, la detección de brechas en recursos humanos y en presupuesto, la optimización de la red y de la contra-referencia, la promoción entre usuarios externos e internos e incentivos no pecuniarios por metas logradas. También se ha indicado la necesidad de proveer recursos en sistemas informáticos, crear un presupuesto único del programa, capacitación del equipo de salud e incentivos hacia los médicos de atención privada o de libre elección para mejorar la cobertura, notificación y registro de los $\operatorname{casos}^{24}$. Las campañas de difusión del programa hacia la comunidad parecen también ser necesarias.

La aplicación del tamizaje para CCU acerca al paciente al reconocimiento de diferentes enfermedades infecciosas transmisibles o no transmisibles como tricomoniasis, sífilis, herpes simplex, gonorrea, vaginosis bacteriana y candidiasis vaginal ${ }^{25}$. En ciertos casos permite también identificar otras patologías tumorales como el cáncer de mama, de endometrio u ovarios. La consulta para el tamizaje permite también abrir el espacio para la educación del paciente u orientarlo en aspectos relativos a educación sexual, planificación familiar, prevención de enfermedades de transmisión sexual y/o estudio de algunas de ellas. En estudios efectuados en otros países de Latinoamérica, una visita reciente al médico ha resultado determinante en la realización del tamizaje cervical ${ }^{21}$. De esta manera, las ventajas de la interacción entre el paciente y el equipo de salud durante el proceso de tamizaje de CCU resultan ser amplias y polivalentes ya que permiten incluir muchos aspectos preventivos en una sola visita.

El Papanicolau ha sido por décadas la piedra angular en el tamizaje para la prevención y el tratamiento del CCU pero sólo puede ser efectivo si hay un sistema bien organizado de seguimiento, diagnóstico y tratamiento. De acuerdo a la OMS, la tarea fundamental del sistema de salud pública, es la aplicación de un programa bien organizado de tamizaje citológico, el que actúa como prevención secundaria luego de que se ha establecido una infección persistente ${ }^{26}$. Un programa de inmunización sólo es posible en países con programas de prevención secundaria bien organizados y que además pueden sostenerlo económicamente.

\section{Prevención primaria a través de vacunas para VPH}

El desarrollo de vacunas para VPH ha permitido reducir el riesgo de infección persistente por subtipos de alto riesgo y de lesiones pre-neoplásicas asociadas a los subtipos contenidos en la vacuna y secundariamente, según la cobertura, a reducir la incidencia de verrugas genitales en la población masculina y femenina ${ }^{27-29}$. Sin embargo, debido al largo período de latencia entre infección y la aparición de CCU, aún no se ha demostrado directamente que la vacuna disminuya el riesgo de $\mathrm{CCU}^{7}$. Por otra parte, las vacunas para VPH carecen de cobertura para subtipos menos prevalentes en los países en desarrollo y existe el 
riesgo de emergencia de estos subtipos no incluidos en ellas como patógenos epidemiológicamente importantes y que reemplacen a los más frecuentes como causas de infección ${ }^{7}$.

La anunciada incorporación de la vacuna VPH en el Programa Nacional de Inmunizaciones en Chile enfrentaría cinco dificultades potenciales y que se refieren al deterioro de la cobertura del tamizaje citológico cervical, a la baja tasa de mortalidad ya alcanzada para el CCU en Chile antes de incorporar la vacuna, a las dificultades progresivas que ha tenido el Programa Nacional de Inmunizaciones en su cobertura en años recientes, a las limitaciones en el análisis de sensibilidad que tienen los estudios de costo-eficacia efectuados en Chile y potencialmente a efectos adversos mayores observados durante el uso de estas vacunas.

\section{Baja cobertura del tamizaje cervical}

Ya se ha comentado sobre el deterioro en las coberturas del tamizaje mediante Papanicolau en la población, lo que es contraproducente para la implementación de la vacunación ya que todos recomiendan que luego de introducir la vacuna se debe continuar este tamizaje en forma periódica y sistemática ${ }^{2,7}$. La continuación y la ampliación del programa de tamizaje es muy importante en un trasfondo de vacunación contra VPH porque la vacuna no previene el desarrollo de lesiones pre-cancerosas o de cáncer en las mujeres ya infectadas por estos virus y tampoco previene la infección por otros VPH que circulen en la población con potencial oncogénico y no cubiertos por la vacuna.

\section{Reducción en la tasa de mortalidad del CCU en Chile y carga de enfermedad}

Queda claramente establecido que los esfuerzos en aumentar la cobertura del tamizaje citológico se asociaron a una reducción de la mortalidad por CCU en Chile y que la mayor tasa observada respecto a los países desarrollados se debe a las dificultades para lograr el $80 \%$ de cobertura establecido como meta inicial. La reducción en la mortalidad implica que es cada vez más caro salvar un año de vida ya que hay progresivamente menos muertes. Actualmente, el CCU ocupa el sexto lugar de mortalidad en mujeres con una tasa ajustada de 5,82 por 100.000 mujeres (Tabla 1). Como carga de enfermedad, expresada como pérdida de años de vida ajustados por discapacidad (AVAD), el CCU ha ido perdiendo importancia a través de los años. Así, el año 1993 el CCU representaba en total 15.628 AVAD perdidos y ocupaba el lugar $\mathrm{N}^{\circ} 15$ entre el total de causas con el $0,9 \%$ del total ${ }^{1}$. En contraste, hacia el año 2004 el CCU representaba 12.860 AVAD perdidos $(0,34 \%$ del total $)$ y ocupaba el lugar $\mathrm{N}^{\circ} 22$. De esta manera y en perspectiva, la vacuna VPH parece introducirse en un escenario epidemiológico de baja incidencia de CCU y que conspiraría en contra de la justificación de su intro- ducción. El mayor peso en la pérdida de AVAD lo tiene la muerte prematura con 9.192 años de vida perdidos por muerte prematura $(71,5 \%)$. La cifra restante para completar los 12.860 AVAD perdidos se explica por la suma de años perdidos con discapacidad (3.668 AVAD perdidos equivalente a $28,5 \%)^{1}$. El peso que tienen las muertes prematuras es relevante en la carga de enfermedad por CCU y una reducción en sus cifras impacta fuertemente en los estudios de costo-efectividad.

\section{Problemas con la cobertura poblacional del Programa Nacional de Inmunizaciones}

A las deficiencias comentadas en la cobertura del tamizaje cervical y de la pesquisa de casos de tuberculosis, se suma un deterioro del PNI para lograr una cobertura adecuada en las vacunas de los primeros años de vida (Figura 2) ${ }^{30}$. Con la excepción de dos vacunas (DPT primer refuerzo y polio primer refuerzo), todas las otras muestran tendencias negativas respecto de su uso, siendo más marcadas la disminución en cobertura con la vacuna BCG y el refuerzo de la vacuna para el sarampión rubéola y parotiditis. Aplicar una vacuna para VPH en años no contemplados en el esquema actual (cuarto y quinto de enseñanza primaria), impone nuevos retos y demandas a

Tabla 1. Mortalidad ajustada en mujeres por diferentes tipos de cáncer el año 2010. Fuente DEIS (referencia 8)

\begin{tabular}{lc|}
\hline Tipo de cáncer & $\begin{array}{c}\text { Tasa de mortalidad ajustada } \\
\text { por } \mathbf{1 0 0} .000 \text { mujeres }\end{array}$ \\
Mama & 12,74 \\
Vesícula & 12,11 \\
Pulmón & $11,4^{*}$ \\
Gástrico & 10,31 \\
Colon & 6,71 \\
CCU & 5,82 \\
Ovario & 4,13 \\
Leucemia & 2,92 \\
Linfoma no Hodgkin & 2,82 \\
Mieloma & 2,06 \\
Recto & 1,96 \\
Endometrio & 1,69 \\
Cáncer de piel no melanoma & 1,16 \\
Melanoma & 1,05 \\
Tiroides & 0,77 \\
Osteosarcoma & 0,55 \\
Linfoma de Hodgkin & 0,25 \\
Anal & 0,20 \\
\hline *Tasa observada, no ajustada. & \\
\hline
\end{tabular}




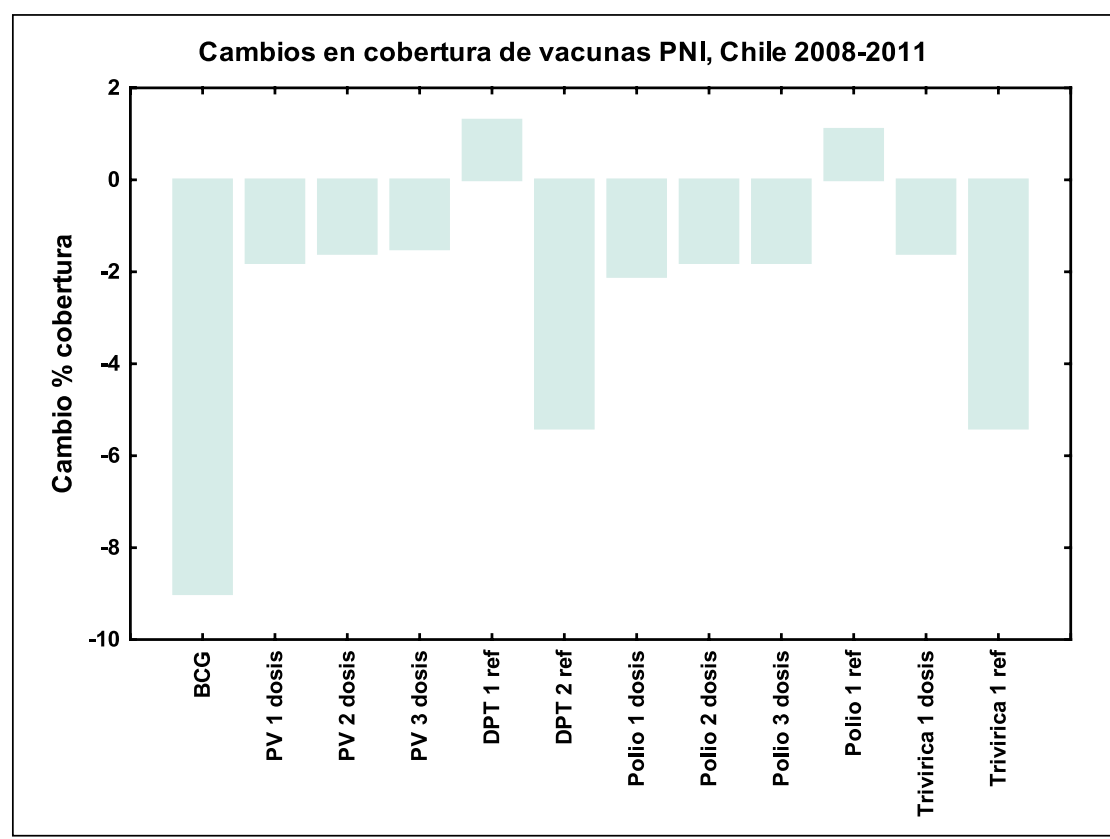

Figura 2. Cambios en la cobertura de algunas vacunas del PNI entre los años 2008-2011. Una cifra negativa indica una baja en la cobertura. PV: vacuna pentavalente en sus diferentes dosis, DPT: vacuna pertussis celular con toxoide diftérico-tetánico en primer y segundo refuerzo, Trivírica: vacuna sarampión, rubéola y parotiditis en su dosis inicial y refuerzo. Fuente Referencia 30.

un programa de inmunización que demuestra importantes debilidades en su habilidad para alcanzar las coberturas necesarias de la población con vacunas de probada eficacia. Se ha argüido que esta baja es secundaria a una menor natalidad lo que no parece ser una explicación válida ya que en tal caso la caída en la cobertura de vacunación en el primer año de vida debería ser similar en todas ellas y no se debería además observar un aumento en la cobertura en ciertas vacunas sobre otras.

\section{Costo de adquisición de las vacunas para VPH y estudios de costo eficacia con algunos supuestos no vigentes}

Las tres dosis de un esquema de vacunación para VPH bordean internacionalmente los US\$ 360, y US\$ 240 en caso de un esquema de dos dosis. Estos precios podrían ser menores en caso de utilizarse el fondo rotatorio de vacunas de la OPS ( $\sim$ US\$ 15 por dosis y US\$ 30 a 45 por esquema $)^{2}$. Para cualquiera de estos valores, un programa de vacuna para VPH es costo-incremental, es decir debe ir asociado a un aumento importante del presupuesto ya que no reduce los costos totales. Se acepta internacionalmente, que si una estrategia de salud permite evitar la pérdida de un AVAD a una cifra $\leq 3$ veces el producto interno bruto per capita, se convierte en una alternativa costo-efectiva razonable a pesar del aumento necesario del presupuesto ${ }^{3}$. Si la adquisición de la vacuna se efectúa a un valor mayor, resulta entonces ser económicamente excesiva y compite con otras necesidades de salud y puede paradójicamente postergar las correcciones al programa de tuberculosis o la misma pesquisa del CCU a través del tamizaje con Papanicolau. Análisis efectuados usando el precio internacional de la vacuna VPH con tres dosis, estimaron un valor entre US\$ 60 y 74 mil por AVAD evitado ${ }^{1}$, cifra $>3$ veces superior al PIB per capita, lo que sin lugar a dudas indica una razón costo/efectividad injustificada en las presentes condiciones.

Sin embargo, los estudios de costo-efectividad son efectuados utilizando diferentes fuentes de información y supuestos que pueden no ser correctos en escenarios epidemiológicos cambiantes. Por ejemplo, el estudio efectuado por el Ministerio de Salud de Chile y publicado el año 2011 utiliza una mayor tasa de mortalidad a la registrada en años recientes con 636 decesos el año 2008 vs 578 el año $2010^{1}$. Asume además una cobertura de vacunación de 95 ó $100 \%$ y un esquema con tres dosis sin necesidad de refuerzo. La existencia de un menor número de decesos hace más difícil demostrar costo-efectividad razonable ya que hay menos AVAD perdidos y si el número de muertes disminuye progresivamente cada año, lo hace aún más difícil en el futuro. Recordemos que el mayor peso de AVAD perdidos se genera por muertes prematuras del CCU invasor más que años vividos con discapacidad. Por otra parte, las tasas de cobertura de vacuna usadas podrían no ser reales ya que no dan cuenta del posible deterioro observado en años recientes de los programas de inmunización en Chile. Finalmente, la persistencia de la inmunidad y la eficacia protectora de la vacuna a través de los años, especialmente con esquemas con dos dosis, es un supuesto que aún no ha sido demostrado y podría suceder que el CCU no se evita sino que sólo se posterga. Si se consideran dosis de refuerzo, los costos aumentarían aún más. Todos estos argumentos indican que los estudios de costo/efectividad discutidos deberían ser revisados con un nuevo análisis de sensibilidad con valores más flexibles y reales, incluyendo los reducidos valores de la vacuna VPH que se pueden obtener a través del Fondo Rotatorio de la OPS (< US\$ 15 por dosis) $)^{2}$. Esto no es solamente un tema académico, sino que necesario desde el punto de vista de la justificación de esta alternativa, la que debiera estar basada en supuestos adecuados e invulnerables. Por ejemplo, es muy probable que gracias a los bajos valores por dosis al aplicar el fondo rotatorio de la OPS, el uso de la vacuna pueda finalmente ser una estrategia costo-efectiva. El costo de un AVAD evitado se reduciría de US\$ 60-74 mil a un valor cercano a los US\$ 9 mil según cálculos del autor, lo que la convertiría en una opción muy costo-efectiva ya que representa $<1$ PIB per capita pero que aún mantiene un margen de error ya que no considera la reducción observada en el número de muertes por CCU en los últimos años ni la necesidad 
de una dosis de refuerzo. Tampoco se han considerado incrementos en la paridad cambiara lo que puede aumentar los costos de adquisición (\$519 en el estudio de costoefectividad versus $\$ 549$ a valor actual). La justificación de esta vacuna en países desarrollados con una baja tasa de mortalidad por CCU es aún más difícil debido al alto costo del esquema y a la necesidad de lograr coberturas perfectas para conseguir relaciones de costo-eficacia aceptables $^{30}$.

\section{Efectos adversos mayores con vacunas $\mathrm{VPH}$}

En algunos países desarrollados como E.U.A. y Países Bajos, la tasa de efectos adversos mayores asociados a la vacuna es superior en 2,5 a 4 veces a la tasa de mortalidad por $\mathrm{CCU}^{31}$. Además, la frecuencia poblacional de efectos adversos en el Reino Unido es muy superior a la observada con otras vacunas comunes al PNI o se ha incrementado notoriamente luego de la introducción de la vacuna VPH como en Australia ${ }^{31}$. Estos efectos adversos mayores han incluido anafilaxis y convulsiones. Otras manifestaciones serias incluyen mielitis transversa, parálisis facial, síndrome de Guillain-Barré, encefalomielitis aguda desmielinizante, síndrome de taquicardia postural, etc. Lamentablemente, el registro de efectos adversos es en la mayor parte de los casos un acto pasivo y parece estar seriamente subestimado. Autores independientes han planteado que se debe analizar en profundidad estos efectos adversos y mejorar su detección ${ }^{31}$.

\section{Otros problemas}

La introducción de la vacuna VPH genera otras interrogantes y/o limitaciones. Entre éstas, la posibilidad de un reemplazo epidemiológico de los subtipos virales hacia variantes no contenidas en la vacuna, la caída del efecto protector a medida que se documentan seguimientos más alejados y deterioros en la cobertura del estudio citológico cervical por una falsa sensación de seguridad en la población. Si todo esto sucede en un contexto en que existen limitaciones importantes del tamizaje por Papanicolau y de limitaciones en alcanzar una cobertura adecuada con la vacuna, se podría potencialmente producir un aumento de la morbilidad y mortalidad en la población susceptible por CCU.

\section{¿Existen mejores alternativas que el Papanicolau para la detección precoz del CCU?}

En los últimos años se han introducido progresivamente métodos moleculares para la detección de la infección de subtipos de alto riesgo de VPH en muestras cervicales. Estas técnicas tienen la ventaja de una mayor sensibilidad que el tamizaje convencional pero una menor especificidad debido a que pesquisan infecciones no persistentes o persistentes no progresivas, además de las infecciones asociadas a neoplasias intra-epiteliales ${ }^{10-12,26}$.
Debido a que las infecciones no persistentes y aquellas persistentes regresivas son más propias de mujeres jóvenes, su aplicación en mujeres $<30$ años determina un sobre-diagnóstico y una demanda exagerada por derivaciones y colposcopias. Meta-análisis comparativos entre el diagnóstico de infección por VPH usando métodos moleculares versus citología convencional indican que en mujeres $>30$ años, estos nuevos métodos permitirían detectar un mayor número de casos de NIE tipo II y III con la misma especificidad que la citología y podrían ser justificados en este segmento ${ }^{10}$. Sin embargo, hasta ahora estas nuevas metodologías se han aplicado y evaluado principalmente en países desarrollados y su costo es mayor que el del Papanicolau. En el escenario nacional, caracterizado por limitaciones en la cobertura del tamizaje tradicional, es difícil plantear su implementación en este momento.

\section{Conclusiones}

El advenimiento de vacunas para el VPH ha permitido incorporar una estrategia de prevención potencialmente primaria para el CCU que se suma a la prevención secundaria lograda con el tamizaje citológico y la detección molecular de la infección por VPH del cáncer cervical. En el pasado y sin vacuna disponible, el uso sistemático de un programa de detección precoz permitió reducir notoriamente la mortalidad por CCU en Chile a pesar de no lograr las coberturas poblaciones deseadas en mujeres de alto riesgo. Los estudios de costo-efectividad para la introducción programática de las vacunas VPH en Chile no son concluyentes debido a varios factores que incluyen la sobreestimación de muertes por esta patología, a las limitaciones para obtener altas coberturas de vacunación además de la reducción que podría producirse en los precios. Así, es probable que al usar el fondo rotatorio de vacunas de la OPS que permite el acceso a vacunas con un menor precio, la inmunización para VPH termine siendo una estrategia costo-efectiva, situación que debería ser confirmada con un nuevo análisis. Algunas presunciones de la decisión actual como la suficiencia de dos dosis en lugar de tres, la protección a largo plazo y la ausencia de un reemplazo hacia subtipos virales no contenidos en la vacuna, podrían no ser necesariamente confirmadas en el futuro. Finalmente, el país está en deuda con el hasta recientemente exitoso programa de tamizaje cervical que muestra un deterioro progresivo en su cobertura en años recientes, a pesar de haber sido la piedra angular del control sobre el CCU en Chile sin necesidad de vacunas. El tamizaje se ha demostrado, además, como una herramienta muy efectiva en el mundo a disminuir la morbilidad y mortalidad por CCU a una fracción de las cifras observadas en Chile al cual se podría agregar 
la detección de los VPH con métodos moleculares en grupos seleccionados de pacientes y luego de resolver las deudas pendientes en salud pública. Parece ser que las discrepancias existentes hace cinco años en torno a esta decisión en Chile continúan estando vigentes lo que atestigua posibles debilidades de esta decisión.

\section{Resumen}

El Ministerio de Salud de Chile ha tomado la decisión de incorporar la vacuna para el virus papiloma humano (VPH) al Programa Nacional de Inmunizaciones (PNI) desde el año 2014 para prevenir el cáncer cérvico-uterino (CCU) a pesar de la existencia de dos documentos discrepantes sobre el tema y a una clara disminución de la mortalidad por CCU en los últimos años gracias al tamizaje citológico cervical. La menor mortalidad por $\mathrm{CCU}$ indica que la carga de enfermedad por esta causa ha disminuido y que los estudios de costo-efectividad efectuados para analizar la incorporación de esta vacuna deben ser revisados incorporando esta nueva información, junto a posibles menores coberturas del PNI como se documenta actualmente, al uso de dosis de refuerzo ya que la eficacia protectora a largo plazo no ha sido demostrada y costos de adquisición reducidos de aplicarse el fondo rotatorio de vacunas de la OPS. Los efectos adversos serios de estas vacunas, en algunos países más frecuentes que la mortalidad por CCU, no han sido adecuadamente analizados aún y parecen estar subestimados. Esta decisión ocurre en medio de un deterioro progresivo en la cobertura del tamizaje lo que pone en riesgo la conquista lograda hasta ahora y atenta contra la incorporación de la vacuna ya que no incluye todos los subtipos de alto riesgo y el riesgo de CCU no desaparece. La decisión de vacunar para el VPH aún requiere un fundamento sólido y no puede ocurrir sin solucionar el problema de la cobertura del tamizaje.

\section{Referencias bibliográficas}

1.- Ministerio de Salud de Chile. Sistematización de la información sobre cáncer cérvicouterino en Chile: Revisión y análisis de estudios de costoefectividad de la vacuna contra VPH. 2011. Disponible en: http://desal.minsal.cl/wp-content/ uploads/2013/09/Sistematizaci\%C3\%B3n.pdf (accedido 2 de enero de 2014).

2.- Comisión Nacional de Vacunas y Estrategias de Vacunación. Chile. Vacuna contra el virus del papiloma humano. Disponible en http://www. siad-sps.cl/file/download/7738 (accedido 2 de enero de 2014).

3.- Abarca K, Valenzuela MT, Vergara R, Luchsinger V, Muñoz A, Jiménez J, et al. Declaración del Comité Consultivo de Inmunizaciones de la Sociedad Chilena de Infectología respecto a la vacuna anti-virus papiloma humano. Septiembre 2008. Rev Chilena Infectol 2008; 25: 428-34.

4.- Cabello F. Potencial prevención del cáncer cérvico uterino en Chile por la vacuna contra virus papiloma humano: más interrogantes que respuestas. Rev Med Chile 2009; 137: 990-3.

5.- Valenzuela MT, O'Ryan M. Réplica. Rev Med Chile 2009; 137: 993-4.

6.- Pisani P, Parkin D M, Bray F, Ferlay J. Estimates of the worldwide mortality from 25 cancers in 1990. Int J Cancer 1999; 83: 18-29.

7.- Van Bogaert L J. Are de currently existing anti-human papillomavirus vaccines appropriate for the developing world? Ann Med Health Sci Res 2013; 3: 306-12.

8.- Departamento de Estadísticas e Información en Salud. Defunciones, mortalidad observada y ajustada por tumores malignos según localización, por región y sexo. Chile 20002010. Disponible en http://www.deis.cl/ defunciones-y-mortalidad-por-causas/ (accedido 30 de diciembre de 2013).

9.- Bosch F X, Burchell A N, Schiffman M, Giuliano A R, de Sanjose S, Bruni L, et al. Epidemiology and natural history of human papillomavirus infections and type-specific implications in cervical neoplasia. Vaccine 2008; 26 (Suppl 10): K1-16.

10.- Pileggi C, Flotta D, Bianco A, Nobile CG, Pavia M. Is HPV DNA testing specificity comparable to that of cytological testing in primary cervical cancer screening? Results of a meta-analysis of randomized controlled trials. Int J Cancer 2013 Dec 3. doi: 10.1002/ ijc.28640. [Epub ahead of print].

11.- Sankaranarayanan R, Nene B M, Shastri S S, Jayant K, Muwonge R, Budukh A M, et al. HPV screening for cervical cancer in rural India. $\mathrm{N}$ Engl J Med 2009; 360: 1385-94.

12.- Leinonen $M K$, Nieminen $P$, Löonberg $S$, Malila N, Hakama M, Pokhrel A, et al. Detection rates of precancerous and cancerous cervical lesions within one screening round of primary human papillomavirus DNA testing: prospective randomised trial in Finland. Br Med J 2012, 345: e7789.

13.- Sepúlveda C, Prado R. Effective cervical citology screening programmes in middleincome countries: the Chilean experience. Cancer Detec Prev 2005; 29: 405-11.

14.- Suárez E, Prieto M, Rojas I, Fernández B, Prado R, Olfos P. Programa Nacional de Cáncer Cérvicouterino. Rev Chil Ginecol Obstet 2001; 66: 480-91.

15.- Donoso E, Cuello M, Villarroel L. Reducción de la mortalidad por cáncer cérvico uterino en Chile, 1990-2003. Rev Chil Obstet Ginecol 2006; 71: 307-12.

16.- Guzmán S, Salas P, Puente R, Hott H, Israel E, Guzmán R. Pesquisa y control del cáncer cérvico-uterino en el Servicio de Salud de Valdivia (1993-2003). Rev Med Chile 2005; 113: 685-92.

17.- Salas P, Guzmán S, Cazor G. Estrategias efectivas para aumentar la cobertura del Papanicolau en el programa de detección del cáncer cérvicouterino. Rev Chil Salud Pública 2005; 9: 12-9.

18.- Valenzuela M T, Miranda A. ¿Por qué no me hago el Papanicolau? Barreras sicológicas de mujeres de sectores populares de Santiago de Chile. Rev Chil Salud Pública 2001; 5(2/3): 75-80.

19.- Lamadrid Alvarez S. Knowledge and fears among Chilean women with regard to the Papanicolaou test. Bull Pan Am Health Org 1996; 30: 354-61.

20.- Serra I, García V, Viñales D, Serra J, Serra L, Zamorano L, et al. Audit of deaths due to cervical cancer in a health service of Santiago. A preliminary analysis. Rev Med Chile 1998; 126: 1010-8.

21.- Soneji S, Fukui N. Socioeconomic determinants of cervical cancer screening in Latin America. Rev Panam Salud Publica 2013; 33: 174-82.

22.- Peña C, Farga V. El difícil camino del control sanitario de la tuberculosis. Rev Chil Enf Respir 2012; 28: 311-8.

23.- Ministerio de Salud de Chile. 20 años del Programa Nacional del Cáncer. 2007. Disponible en http://web.minsal.cl/portal/url/it em/71716b3c3eed775ce04001011f0151ca.pdf 
(accedido 30 de diciembre de 2013).

24.- Lanza S, Sepúlveda C, Olate M, Espejo C. Aplicación de metodología de marco lógico para el análisis del programa nacional de pesquisa y control del cáncer cérvicouterino en Chile. Rev Chil Obstet Ginecol 2010; 75: 294-9.

25.- Otárola C, Briceño J, Bahamondes M I, Muñoz R, Lorca M. Frecuencia de Trichomonas vaginalis detectadas mediante Papanicolau en cuatro Servicios de Salud, 1997-2002. Rev Chil Obstet Ginecol 2005; 70: 3-7.

26.- Corusić A, Skrgatić L, Mahovlić V, Mandić V, Planinić P, Karadza M. Cervical cancer as a public health issue: What next? Coll Antropol 2010; 34: 301-7.

27.- Ali H, Donovan B, Wand H, Read T R, Regan D G, Grulich A E, et al. Genital warts in young Australian five years into national human papillomavirus vaccination programme: national surveillance data. Br Med J 2013; 346: f2032.

28.- Read T R, Hocking J S, Chen M Y, Donovan B, Bradshaw C S, Fairley C K. The near dissapearence of genital warts in young women 4 years after commencing a national human papillomavirus (HPV) vaccination programme. Sex Transm Infect 2011; 87: 544-7. 29.- Leval A, Herweijer E, Arnheim-Dahlström L,
Walum H, Frans E, Sparén P, et al. Incidence of genital warts in Sweden before and after quadrivalent human papillomavirus vaccine availability. J Infect Dis 2012; 206: 860-6.

30.- Diario La Tercera. Ministerio de Salud ordena estudio tras baja general en vacunación a nivel nacional. 2 de septiembre del 2013, página 10. Disponible en http://www.papeldigital.info/ 1t/2013/09/02/01/paginas/010.pdf (accedido 23 de enero de 2014).

31.- Tomljenovic L, Shaw C A. Human papillomavirus (HPV) vaccine policy and evidence-based medicine: Are they at odds? Ann Med 2013; 45: 182-93. 